\title{
Detection of a narrow-band noise as \\ a function of its interaural correlation'
}

\author{
W. A. WILBANKS AND JOHN K, WHITMORE 2 \\ UNIVERSITY OF MISSISSIPPI
}

The detectability of a narrow-band noise is shown to be a function of the interaural correlation of both the signal and the masker. The maximal release from masking occurs when the addition of the signal results in a reduction in the correlation of the masking noise. The results show that people are far more sensitive to a decrease in the correlation between the stimuli at the two ears than to an increase.

Research on the effects of varying the interaural correlation of a masking noise on the detectability of binaural and monaural signals has clearly shown that the magnitude of the correlation between the temporal events at the two ears is a dominant variable affecting binaural detection (e.g., Robinson \& Jeffress, 1963; Whitmore \& Wilbanks, 1965a, b; Wilbanks \& Whitmore, 1967). The present study extends this work to the detection of a narrow-band noise as a function of its interaural correlation, as well as of the correlation of the masker. Method

The signal was a $135-\mathrm{Hz}$ narrow-band noise centered at $250 \mathrm{~Hz}$. Two noise generators were used to obtain signals with interaural correlations of +1.00 , $+.81,+.56,+.25,+.06$, and zero. The unity correlation was obtained by using a single noise source for both ears. The zero correlation was achieved by using independent noise generators, one for each ear. Correlations between unity and zero were obtained by mixing the outputs of the two noise sources in the channel to one ear, with the noise at the other ear being supplied by only one of these generators. 3 The duration of the signal $(200 \mathrm{msec})$ was controlled by an Iconix electronic counter that triggered a GrasonStadler switch, gating the noise with a $25 \mathrm{msec}$ on-off time. The spectral level of each signal, measured at $250 \mathrm{~Hz}$, was adjusted to yield a detection score of $\mathrm{d}_{\mathrm{s}}=1.50$ for each experimental condition.

The signals were partially masked by continuous wide-band noise, cut off at the lower limit by the response of the earphones (TDH-39), and at the upper end by a $3000 \mathrm{~Hz}$ low-pass passive filter. The spectral level of the masker corresponded to approximately +33 dB re .0002 microbar per cycle. Detection was studied for each signal correlation under two conditions of masker correlation, +1.00 and zero. 4

Detection was also studied under three additional conditions: (1) masker +1.00 , signal monaural-NO-SM; (2) masker uncorrelated, signal monaural-NU-SM; and (3) masker monaural, signal monaural-NM-SM.

Masked thresholds, defined as the signal level in $\mathrm{dB}$ for $\mathrm{d}_{\mathrm{S}}=1.50$, were obtained on three experienced Ss by means of the fixed-interval observation experi- ment (FIOE) as described by Egan, Greenberg, \& Schulman (1961). The listeners used a five-category rating scale to indicate the likeness of the momentary input to the noise. 5 The rating-scale data yielded receiver-operating-characteristic (ROC) curves, from which measures of detection, $d_{s}$, were obtained. Threshold signal levels were determined by the method described by Jeffress (1964), and by Wilbanks \& Whitmore (1967).

\section{Resulfs and Discussion}

The results are shown in Fig. 1. Here the ordinate is the signal level in $d B$ for $d_{S}=1.50$, the abscissa is the interaural correlation for the signal, and the parameter is the correlation for the masker. Inspection of the data shows that when the masker is uncorrelated (NU), increasing the correlation of the signal from zero to +1.00 results in an improvement in detection on the order of $2 \mathrm{~dB}$. With a correlated masker, however, detection improves by some $8 \mathrm{~dB}$ as the correlation of the signal is reduced from unity to zero. 6 An uncorrelated signal (SU) is about $14 \mathrm{~dB}$ more detectable with a correlated masker than with an uncorrelated masker, and about $16 \mathrm{~dB}$ better than the purely monaural condition (NM-SM). With a monaural signal, the addition of uncorrelated noise at the non-signal ear has a negligible effect on detection. The addition of correlated noise, on the other hand, results in an improvement on the order of $12 \mathrm{~dB}$. The difference between NO-SM and NM-SM here is about $2 \mathrm{~dB}$ more than that found by Wilbanks \& Whitmore (1967) for a monaural $250-\mathrm{Hz}$ tonal signal.

Two interesting models have been advanced to ex-

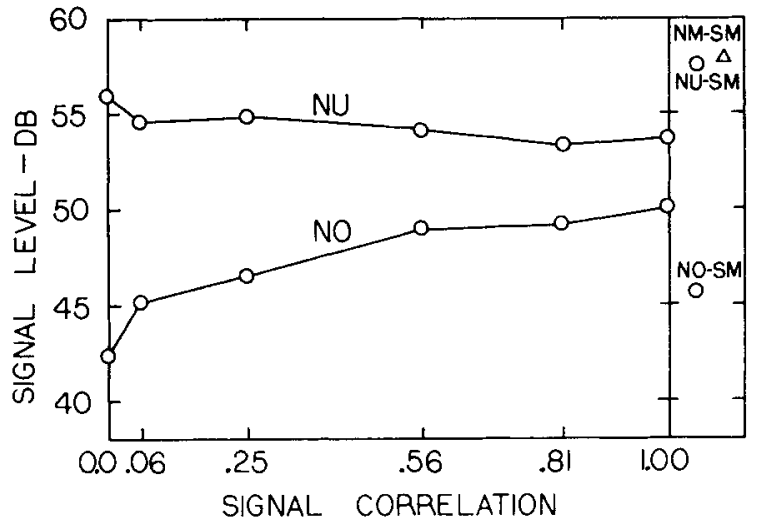

Fig. 1. Signal level in $d b$ for $d_{s}=1.50$ as a function of signal correlation. The parameter is the correlation of the masker. 
plain and organize data obtained from binaural masking experiments, the Jeffress "vector" model (1956, 1965) and Durlach's (1963) equalization-cancellation model. The Jeffress model has somewhat more appeal for the writers in that it subsumes both binaural masking and localization phenomena under one set of principles, and appears to be more amenable to formulation in terms of physiological mechanisms. In brief, the Jeffress hypothesis proposes that the basis for improved detection under binaural listening conditions is the change in the correlation between the stimuli at the two ears when the signal is added and, consequently, the change in the correlation between neural events subsequent to the cochlea. The results of the present study show that release from masking is maximal when the addition of the signal results in a reduction in the correlation of the masker. The greatest reduction occurs when an uncorrelated signal is added to a correlated masker, and this is the condition where the signal was most detectable (NO-SU). The small improvement (2 dB) which occurs when the addition of the signal results in an increase in the correlation of the masker (NU-SO) indicates that people are far more sensitive to a decrease in the interaural correlation of the stimuli at the two ears than to an increase.

The notion that the basis for improved detection is the reduction in synchrony between the inputs to the detection mechanism is consistent with electrophysiological knowledge. One is immediately reminded of recent studies of the olivary nucleus (e.g., Moushegian, Rupert, \& Whitcombe, 1964) where cells are found whose activity is related to asynchrony of stimulation from the two ears (a "not-AND gate"), i.e., related to a decrease in the correlation of neural firing between the ears. The results of the present study would indicate that an appropriate black-box model would be one which viewed the detection mechanism as a correlational detector that is primarily sensitive to a reduction in synchrony of the temporal events which constitute the input to the model.

\section{References}

Durlach, N. I. Equalization and cancellation theory of binaural masking-level differences. J. Acoust. Soc. Amer., 1963, 35, 1206-1218.

Fgan, J. P., Greenberg, G. Z., \& Schulman, A. I. Interval of time uncertainty in auditory detection. J. Acoust. Soc. Amer.. 1961, 33, 771-778.

Jeffress, L. A. Stimulus-oriented approach to detection. J. Acoust. Soc. Amcr., 1964, 36, 766-774.
Jeffress, L. A. Masking and binaural phenomena. Univ. Texas Def. Res. Lab. Rep., No. 245, 1965; also personal communication. Jeffress, L. A., Blodgett, H. C., Sandel, T. T., \& Wood, C. L. Masking of tonal signals. J. Acoust. Soc. Amer., 1956, 28, 416426.

Jeffress, L. A., \& Robinson, D. E. Formulas for the coefficient of interaural correlation for noise. J. Acoust. Soc. Amer., 1962, $34,1658(\mathrm{~L})$.

Licklider, J. C. R., \& Dzendolet, E. Oscillographic scatterplots illustrating various degrees of correlation. Science, 1948, 107 , 121-124.

Moushegian, G., Rupert, A., \& Whitcombe, M. A. Brain stem neuronal response patterns to monaural and binaural tones. J. Neuro* physiol., 1964, 27, 1174-1191.

Robinson, D. E., \& Jeffress, L. A. Effect of varying the interaural noise correlation on the detectability of tonal signals. J. Acoust. Soc. Amer., 1963, 35, 1947-1952.

Whitmore, J. K., \& Wilbanks, W. A. Interaural noise correlation and monaural signal detection. J. Acoust. Soc. Amer., 1965a, 37, 1179 (A).

Whitmore, J. K., \& Wilbanks, W. A. Interaural noise correlation and detection of low-frequency monaural signals, J. Acoust. Soc. Amer., 1965b, 38, 929 (A).

Wilbanks, W, A., \& Whitmore, J. K. Detection of monaural signals as a function of interaural noise-correlation and signal frequency. J. Acoust. Soc. Amer., 1967, in press.

\section{Notes}

1. The principal results of this study were presented to the 70 th Meeting of the Acoustical Society of America, Nov, 1965, St. Louis, Mo. (J. Acoust. Soc. Amer., 1965, 38, 929).

2. Currently, a National Institute of Mental Health Post-doctoral Research Fellow to the Department of Psychology, University of Texas.

3. This way of varying the interaural noise-correlation has been thoroughly discussed by Licklider and Dzendolet (1948), and by Jeffress and Robinson (1962), who point out that it involves reducing the correlation by decreasing the amount of noise variance common to both ears, as in an analysis of variance approach, rather than reducing the correlation by introducing error variance at the two ears, as in a product-moment correlation.

4. In all, four noise generators were used: two to obtain the signal correlations, and two to obtain the masker correlations. Since the signal and the masker were always obtained from independent sources, the phase relation between signal and masker was random for all values of signal and masker correlation.

5. The program of training was similar to the one outlined by Egan, et al (1961). It differed in that there was no attempt to utilize "hit" information, $P(y \mid s n)$, in the establishment of criteria. The observers were trained until the "false-alarm" rate, $P(y \mid n)$, for any given cut-off point on the rating-scale was stable across the various interaural conditions. In other words, the subjects were trained to be Neyman-Pearson observers.

6. The difference in favor of NO-SO over NU-SO (about 4 db) requires comment. Since signal and masker were obtained from independent sources, the phase relation between them was random. This would make NO-SO antiphasic relative to NU-SO, and some release from masking would be expected. 